\title{
Discrepancies in the diagnosis of methicillin resistant Staphylococcus aureus among different hospitals in Lahore, Pakistan
}

Romeeza T, Nadeem A, Nauman J, Afia A and Kunwal R

Department of Immunology, University of Health Sciences Lahore, Pakistan

\begin{abstract}
Introduction: Staphylococcus aureus is a pathogen causing a wide range of infections. It changes its genome under the selection pressure of antibiotics. Methicillin resistance is commonly determined by Kirby-Bauer disc diffusion method which has low sensitivity and specificity. Thus, anti PBP-2a latex agglutination test was used to confirm the identity of those labeled as MRSA. Materials and Methods: One hundred bacterial samples characterized as MRSA according to Kirby-Bauer diffusion method were collected from different hospitals in Lahore. Results: Biochemical characterization of these MRSA isolates revealed that only 64 (64\%) were actually S. aureus while the other 36 isolates (36\%) were coagulase negative staphylococci. Further serological characterization revealed that among the 64 isolates of S. aureus, 50 (78\%) were MRSA while 14 (22\%) were MSSA. Conclusion: Therefore, overall, 50\% of the bacterial strains were misidentified as MRSA. Misdiagnosis of antibiotic resistance may lead to an inappropriate prescription of medicines to these patients.
\end{abstract}

KEY WORDS: MRSA, MSSA, Staphylococcus epidermidis, Agglutination

\section{INTRODUCTION}

Staphylococcus aureus is a member of the normal flora of skin and anterior nasal vestibules. ${ }^{1}$ It is an opportunistic pathogen and one of the leading causes of nosocomial and community acquired infections. ${ }^{2}$ It is well established that it can escape from various defensive immune mechanisms of the body. ${ }^{3,4}$ It causes a wide range of infections such as abcesses, bacteremia, central nervous system infections, endocarditis, osteomyelitis, pneumonia, urinary tract infections, chronic lung infections associated with cystic fibrosis, food poisoning, scalded skin syndrome, and toxic shock syndrome. ${ }^{5}$

In 1960, methicillin was developed to treat $\mathrm{S}$. aureus infections because it was the time when $\mathrm{S}$. aureus became resistant to penicillin. However, within a year methicillin resistant $\mathrm{S}$. aureus (MRSA) was also reported in Southern England. ${ }^{1}$ It was found that methicillin resistant gene is encoded by chromosomal mecA gene. The mecA gene encodes for a novel penicillin binding protein, called PBP-2a, instead of normal penicillin binding proteins. Active site of PBP-2a blocks binding of all B-lactams which allows transpeptidation of S. aureus cell wall and therefore, S. aureus contin-

Corresponding author;

Nadeem Afzal

Department of Immunology.

University of Health Sciences,

Lahore, Pakistan

E-mail address: aann30@hotmail.com ues to survive. Incorporation of mecA confers resistance not only to the methicillin but also against all other B-lactams including cephalosporins. ${ }^{1,6}$ There is evidence that mecA gene had been transferred from Staphylococcus epidermidis to $\mathrm{S}$. aureus. ${ }^{7,8}$

In Asian countries like Japan, Korea and China the incidence of MRSA has reached more than $70 \% .{ }^{9}$ In order to control these infections, it is important to screen MRSA samples accurately and as early as possible. Depending upon the screening results appropriate antibiotic treatment can be prescribed and appropriate precautions can be adopted to prevent the spread of resistant pathogens in the hospitals and community. It is believed that screening methods should be highly sensitive, specific, inexpensive and rapid..$^{10,11}$ It is documented that for the detection of MRSA PBP-2a latex agglutination test is simple, rapid, inexpensive and a sensitive screening method. Further this technique has been proven to be more sensitive than oxacillin screen agar method which is used in routine for the detection of MRSA. ${ }^{12}$

\section{MATERIALS AND METHODS}

A cross-sectional descriptive study involving one hundred isolates of methicillin resistant Staphylococcus aureus were collected by non-probability and convenience sampling technique from various public and private sector clinical diagnostic laboratories in Lahore, Pakistan that included Sheikh Zayed Hospital, Ittefaq Hospital, Fatima Memorial Hospital, Shaukut Khanum Hospital, Children Hospital and Chughtai's Laboratory. The sample collection was done during 
the period of February to May 2008. These bacterial isolates were obtained from various sites, such as pus, blood, sputum, tracheal aspirates, etc. The nature of these isolates was reconfirmed by Gram staining, mannitol fermentation, coagulase and DNase production.

Reconfirmation of methicillin resistance: Known isolates of MRSA were reconfirmed by PBP-2a latex agglutination kit (Oxoid). Extraction reagent 1 (four drops) and sufficient bacterial growth (filling $5 \mu \mathrm{l}$ bacterial loop) were added in an Eppendorf tube to make homogeneous suspension. It was placed in a boiling water bath (over $95{ }^{\circ} \mathrm{C}$ ) for three minutes and then allowed to cool at room temperature. Extraction reagent 2 (one drop) was added in the same eppendorf and mixed well. Mixture was centrifuged (3,000 rpm) for five minutes and supernatant was used for the test. Kit is provided with two bottles of latex particles i.e. test latex and control latex. On the test card one drop of test latex and control latex was added to the respective circles. Supernatant was added on test latex and control latex and mixed thoroughly with a separate mixing stick for three minutes and agglutination was noted. Results were interpreted according to following three possibilities.

1. Agglutination in test latex but not in control latex within 3 minutes-----MRSA

2. Agglutination in neither test latex nor control latex within 3 minutes----MSSA

3. Agglutination in control latex but not in test latex within 3 minutes--Intermediate*

*Isolates showing intermediate results were tested again with fresh cultures and results were noted.

Data analysis: Data was entered and analyzed using Excel 2003. Frequencies and percentages were calculated.

\section{RESULTS}

According to the results of biochemical characterization (catalase, coagulase, DNase, and mannitol fermentation tests) 64 isolates (64\%) were found to be $S$. aureus, while 36 isolates (36\%) were coagulase negative staphylococci (Table I). Among the 64 confirmed S. aureus isolates (Table I), 50 (78\%) were found to be methicillin resistant and 14 (22\%) were methicillin sensitive by anti PBP-2a latex agglutination (Table II). Therefore, overall 50\% of staphylococcal bacterial strains were misdiagnosed as MRSA.

Table I: Reconfirmation of S. aureus by different biochemical tests

$\begin{array}{ll}\text { Isolates } & \text { No. (\%) } \\ \text { Staphylococcus aureus } & 64(64) \\ \text { Coagulase negative staphylococci } & 36(36)\end{array}$

Total 100
Table II: Reconfirmation of MRSA by anti PBP-2a latex agglutination*

$\begin{array}{ll}\text { Isolates } & \text { No. (\%) } \\ \text { Staphylococcus aureus } & 64(64) \\ \text { Coagulase negative staphylococci } & 36(36)\end{array}$

Total

100

*Based on the confirmed isolates of S. aureus given in table I.

\section{DISCUSSION}

Prevalence of MRSA has been reported to be up to $42 \%$ in Pakistan. ${ }^{13,14}$ It has been suggested that inappropriate use of antibiotics is one of the triggering factors for the emergence of antibiotic resistant bacteria. ${ }^{15}$ Further resistant organisms evolve and spread at comparatively high rate in those settings where practice of antibiotic prescription is more. ${ }^{16}$

It is reported in the present study that 36 (36\%) isolates that were declared as MRSA by different laboratories were found coagulase negative staphylococci on routine biochemical characterization in our laboratory. Similar observation had been reported by Drew and coworkers that in routine practice many MRSA are actually coagulase negative Staphylococcus epidermidis that are misidentified. S. epidermidis is part of normal flora of skin and contaminates clinical samples if sample collection is not done properly. Methicillin resistance is also a common phenomenon in S. epidermidis. ${ }^{17}$

We found by anti PBP-2a latex agglutination technique that among 64 confirmed isolates of $\mathrm{S}$. aureus, 50 isolates $(78 \%)$ were resistant to methicillin while 14 isolates $(22 \%)$ of $\mathrm{S}$. aureus were actually sensitive to methicillin but had been wrongly declared as MRSA by Kirby-Bauer disc diffusion method in different laboratories. It is well established that disc diffusion method has low sensitivity and specificity as compared to other methods used for the detection of antibiotic resistance. In addition antibiotic susceptibility testing by disc diffusion method may also fail to characterize MRSA strains that exhibit heterogeneous resistance. Heterogeneous resistance is defined as the presence of small resistant colonies of bacteria in the growth inhibition zone of an antibiotic. ${ }^{18}$ Overall, it is found in the present study that $50 \%$ of isolates were misidentified as MRSA; they were either coagulase negative staphylococci or MSSA.

Appropriate screening of MRSA isolates by rapid, specific and sensitive methods is essential for therapeutic and epidemiological purposes. PCR is considered a gold standard method for the detection of mecA gene but it is expensive and labour intensive and not all laboratories could have the facilities to perform this assay. Anti PBP-2a latex agglutination is a reliable and 
rapid diagnostic test that can also be used as an alternative to mecA gene detection by PCR. But there are reports where mecA negative strains have been declared as methicillin resistant by this technique and vice versa. These findings could be either due to non functional mecA gene or PBP-2a protein. ${ }^{18}$

Anti PBP-2a latex agglutination test is performed on fresh ( 24 to 48 hours old) isolated colonies on different media like Columbia agar with $5 \%$ sheep blood, tryptone soy agar with 5\% sheep blood, and MuellerHinton agar. Results of anti-PBP-2a latex agglutination are visible within 20 minutes and can be visualized with an unaided eye. ${ }^{19,20}$ The sensitivity of the Oxoid PBP2' test was found to be $98.5 \%$ and the specificity was $100 \% .19$ It has been proven that results of monoclonal PBP-2a antibody based latex tests correlate $100 \%$ with the detection by genotypic methods. Furthermore, agglutination method is rapid, specific and sensitive. ${ }^{21,22}$ Earlier detection of methicillin resistance in critical cultures like blood and sterile fluids may allow the clinicians to change or initiate antibiotic regimen. ${ }^{23}$ Oxacillin disc diffusion test has been reported as least reliable method for detecting MRSA. ${ }^{24-27}$

In common practice, because of the misdiagnosis of patients infected with MSSA as MRSA, they are treated with vancomycin. It has also been reported that in countries where rate of MRSA infection is high, prophylactic treatment with vancomycin is often initiated. It is well documented that vancomycin also eliminates the normal flora and that may lead to opportunistic infections. It should be remembered that at the moment misuse of vancomycin may produce vancomycin resistance and thus narrowing treatment options. In addition vancomycin is also an expensive and toxic drug. Hence, earlier detection of MRSA by PBP-2a latex agglutination test may restrict the use of vancomycin to the patients who really need it. ${ }^{28,29}$

PBP-2a detection by latex agglutination is much simpler and rapid test for the screening of methicillin resistance. It is much easier to perform than genotypic methods and gives clear visible results within minutes. Rapid detection of MRSA may allow earlier initiation of treatment and as a result morbidity and mortality rates can be decreased. Earlier detection may not only reduce pharmacy costs and hospital expenditure but can also provide better infection control.

\section{ACKNOWLEDGEMENTS:}

This work was supported by a grant from the University of Health Sciences, Lahore Pakistan

DISCLAIMERS: None

\section{REFERENCES}

1. Grundmann H, Aires de-Sousa M, Boyce J, Tiemersma E. Emergence and resurgence of methicillin-resistant Staphylococcus aureus as public-health threat. Lancet 2006; 368:874-85

2. Feng Y, Chen CJ, Su LH, Hu S, Yu J, Chiu CH. Evolution and pathogenesis of Staphylococcus aureus: lessons learned from genotyping and comparative genomics. Microbiol Rev 2008; 32:2337

3. Nizet V. Understanding how leading bacterial pathogens subvert innate immunity to reveal novel therapeutic targets. J Allergy Clinical Immunol 2007;120:13-23

4. Langley R, Wines B, Willoughby N, Basu I, Proft T, Fraser JD. The staphylococcal superantigen-like protein 7 binds IgA and complement $\mathrm{C} 5$ and inhibits IgA-FcaR1 binding and serum killing of bacteria. J Immunol 2005; 174:2926-33

5. Gill SR, Fouts DE, Archer GL, et al. Insights on evolution of virulence and resistance from the complete genome analysis of an early methicillinresistant Staphylococcus aureus strain and a biofilm-producing methicillin-resistant Staphylococcus epidermidis strain. J Bacteriol 2005; 187:2426-38

6. Lowy FD. Antimicrobial resistance: the example of Staphylococcus aureus. J Clin Invest 2003; 111:1265-73

7. Deurenberg RH, Stobberingh EE. The evolution of Staphylococcus aureus. Infect Genet Evol 2008; 8:747-63

8. Deurenberg RH, Vink C, Kalenic S, Friedrich AW, Bruggeman CA, Stobberingh EE. The molecular evolution of methicillin-resistant Staphylococcus aureus. Clin Microbiol Infect 2007; 13:222-34

9. Ko KS, Le JY, Suh JY, et al. Distribution of major genotypes among methicillin-resistant Staphylococcus aureus clones in Asian countries. J Clin Microbiol 2005; 43:421-26

10. Kunori T, Cookson B, Roberts JA, Stone S, Kibbler C. Cost effectiveness of different MRSA screening methods. J Hosp Infect 2002; 51:189-200

11. Stoakes L, Reyes R, Daniel J, et al. Prospective comparison of a new chromogenic medium, MRSASelect, to Chromagar MRSA and mannitolsalt medium supplemented with oxacillin or cefoxitin for detection of methicillin-resistant Staphylococcus aureus. J Clin Microbiol 2006; 44:637-39

12. Chapin KC, Musgung MC. Evaluation of penicillin binding protein $2 \mathrm{a}$ latex agglutination assay for identification of methicillin-resistant Staphylococcus aureus directly from blood cultures. J Clin Microbiol 2004; 42:1283-84

13. Siddiquei GM, Kramat AM, Hannan A. Methicillin resistant Staphylococcus aureus: A study of PAF Hospital Sargodha. Pak J Pathology 1999; 10:26-8

14. Ali AM, Abbasi SA, Arif S, Mirza IA. Nosocomial infections due to methicillin resistant Staphylococcus aureus in hospitalized patients. Pak J Med Sci 2007; 23:593-96 
15. Chinedum IE. Microbial resistance to antibiotics. Afr J Biotechnol 2005; 4:1606-11

16. Mirza SH. Minimising antibiotic resistance-is there a way forward? Infect Dis J 2007; 7:5-9

17. Drew WL, Barry AL, O’ Toole R, Sherris JC. Reliability of the Kirby-Bauer disc diffusion method for detecting methicillin-resistant strains of Staphylococcus aureus. Appl Microbiol 1972; 24:240-47

18. Babay HH. Comparison of susceptibility testing methods for the detection of methicillin/ oxacillin resistance in Staphylococcus aureus. Kuwait Med J 2006; 38:198-02

19. Oxoid penicillin binding protein (PBP2') latex agglutination test [online]. Available at: www. oxoid.com/UK/blue/prod_detail/prod_detail.asp ?pr=DR0900\&c $=$ UK\&lang $=$ EN\&minfo $=Y$

20. Chapin KC, Musgung MC. Evaluation of penicillin binding protein $2 a$ latex agglutination assay for identification of methicillin-resistant Staphylococcus aureus directly from blood cultures. J Clin Microbiol 2004; 42:1283-84

21. Sakoulas G, Gold HS, Venkataraman L, Degirolami PC, Eliopoulos, Qian Q. Methicillin-resistant Staphylococcus aureus: Comparison of susceptibility testing methods and analysis of mecApositive susceptible strains. J Clin Microbiol 2001; 39:3946-51

22. Griethuysen AV, Pouw M, Leeuwen NV, et al. Rapid slide latex agglutination test for detection of methicillin resistance in Staphylococcus aureus. J Clin Microbiol 1999; 37:2789-92

23. Miller MB, Meyer H, Rogers E, Gilligan P. Comparison of conventional susceptibility testing, penicillin-binding protein $2 a$ latex agglutination testing, and mecA real-time PCR for detection of oxacillin resistance in Staphylococcus aureus and coagulase-negative Staphylococcus. J Clin Microbiol 2005; 43:3450-52

24. Cavassini M, Wenger A, Jaton K, Blanc DS, Bille J. Evaluation of MRSA-Screen, a simple anti-PBP $2 a$ slide latex agglutination kit, for rapid detection of methicillin resistance in Staphylococcus aureus. J Clin Microbiol 1999; 37:1591-94

25. Chambers HF. Detection of methicillin resistant staphylococci. Infect Dis Clin N Am 1993; 7:425-33.

26. Knapp CC, Ludwig MD, Washington JA, Chambers HF. Evaluation of the Vitek GPS-SA card for testing of oxacillin against borderline-susceptible staphylococci that lack mec. J Clin Microbiol 1996; 34:1603-05

27. Unal S, Werner K, DeGirolami P, Barsanti F, Eliopoulos G. Comparison of tests for detection of methicillin-resistant Staphylococcus aureus in a clinical microbiology laboratory. Antimicrob Agents Chemother 1994; 38:345-47

28. Hart I. A test for early detection of MRSA: clinical benefits and financial savings [online]. Available at www.cli-online.com/products/microbiology/ bacteria/clinical-laboratory/a-test-forthe-early-detection-of-mrsa-clinical-benefitsand-financial-savings/index.html
29. Enright MC. The evolution of a resistant pathogen- the case of MRSA. Curr opin Pharmacol 2003; 3:474-79 